\title{
A Note on the Extensions of Eratosthenes' Sieve
}

\author{
A.R. QUESADA and B. VAN PELT
}

\begin{abstract}
Given $k \in \mathbb{N}$, let $S_{k}$ denote the set of natural numbers relatively prime to the first $k$ primes. The $k$-extension of the Sieve of Eratosthenes, recently found, provides a set of rules that govern the positions in $S_{k}$ of the multiples of the elements of $S_{k}$. In this paper we provide an alternative approach to the $k$-extension which yields an easier implementation in parallel processing. In addition it is shown that, with an appropriate layout of the set $S_{k}$, the rules governing the sieving process can be made similar to those in the original sieve.
\end{abstract}

Keywords and Phrases. Prime numbers, sieve, tables of primes, algorithms.

\section{INTRODUCTION}

The needs of modern cryptography, fueled by an increase in electronic transmission of information, and the need for computer-testing

1991 Mathematics Subject Classification: 11A41, 11-04, 11 Y16

Servicio publicaciones Univ. Complutense. Madrid, 1996. 
algorithms, explains in part the resurgence of activity in the area of primality that we have witnessed in the last twenty five years. The search for efficient algorithms to generate large tables of prime numbers has produced excellent new results from, among others, Mairson [3], Pritchard [4] and Bengelloun [1]. It has also produced noticeable improvements of traditional algorithms like Eratosthenes' Sieve.

The Sieve of Eratosthenes is probably the best known way to generate the table of all prime numbers less than a given natural number $N$. The reason for this is that, despite its simplicity, the Sieve is reasonably efficient. The primes on an initial set of candidates $S=\{2,3, \ldots, N\}$ are found iteratively by first crossing out all the multiples of 2 larger than 2 in $S$. Then, in each subsequent step, the multiples of the smallest remaining number $p$ (larger than $p$ ) in $S$, not previously considered, are crossed out. Marking the multiples of $p$ can be accomplished simply by counting, since they are located $p$ units apart starting at $p$. This process, of "sieving" the composite numbers in $S$, continues until $p^{2}>N$.

This classic algorithm, as described above, can be readly improved by first letting the initial set be the subset $S_{1}$ of all the odd numbers in $S$; in addition, we can mark the multiples of $p$, still $p$ units apart in $S_{1}$, starting at $p^{2}$. We call this the first extension of the Sieve of Eratosthenes; and we remark that, quite often, the modern-day Sieve of Eratosthenes is identified with this first extension [2].

In 1988, X. Luo [7] achieved the second extension by sieving an initial set $S_{2}$, obtained from $S$, that was devoid of both the multiples of 2 and 3. In 1991, A. Quesada [5] obtained the third extension by further removing the multiples of 5 from the sieving set $S$. Finally, in 1993, Quesada [6] developed the generalized $k$-th extension where the initial set of candidates $S_{k}$ is obtained from $S$ by removing the multiples of the first $k$ primes in $S$. In each subsequent extension, the reduction of the size of the new initial set produces a change in the position of the remaining elements in the set. As a result, the positions of consecutive multiples of any given number $p \in S_{k}$ are no longer $p$ units apart. Instead, for each $p$ in $S_{k}$ a constant finite set of differences between consecutive multiples of $p$ was found. Then the positions of the multiples of $p$ in $S_{k}$ were easily obtained by adding cyclically the elements of this finite set to the position of $p$. As the size of $k$ increases, so does the size of this set of differences, making the calculations needed 
for sieving the $k$-extension too cumbersome to be done by hand. In this paper we provide an alternative approach to the $k$-extension which yields an easier implementation in parallel processing. In addition it is shown that, with the proper layout of the sieving set, the rules governing the sieving process can be made almost as simple as those in the original sieve.

\section{NOTATION AND BASIC DEFINITIONS}

Let $p_{1}, p_{2}, \ldots p_{i}, \ldots$, denote the sequence of prime numbers, and let $\pi_{k}=\prod_{i=1}^{k} p_{i}, k \in \mathbb{N}$. The initial set is defined as $S_{k}=\{x \in \mathbb{N}$ : $\left.\left(x, \pi_{k}\right)=1\right\}$. We denote by $C_{k}$ the subset of elements of $S_{k}$ less than $\pi_{k}$; that is, $C_{k}=\left\{c \in \mathbb{N}: c<\pi_{k},\left(c, \pi_{k}\right)=1\right\}$. We let $m_{k}$ stand for the cardinality of $C_{k}$ which can be easily obtained from the Euler totient function of $\pi_{k}$; i.e., $m_{k}=\left|C_{k}\right|=\phi\left(\pi_{k}\right)=\prod_{i=1}^{k}\left(p_{i}-1\right)$.

Proposition 1. Let $C_{k}=\left\{e_{1}, e_{2}, \ldots, e_{m_{k}}\right\}$, where $e_{i}<e_{j}$ for $i<j$. The following statements hold:

a) $S_{k}=\left[e_{1}\right] \cup\left[e_{2}\right] \cup \ldots \cup\left[e_{m_{k}}\right]$, where $\left[e_{i}\right]=\left\{x \in S_{k}: x \equiv e_{i}\left(\bmod \pi_{k}\right)\right\}$ for $1 \leq i \leq m_{k}$, and $\left[e_{i}\right] \cap\left[e_{j}\right]=\phi$ for $i \neq j$.

b) For $1 \leq i \leq m_{k},\left[e_{i}\right]=\left\{e_{i}, e_{i}+\pi_{k}, \ldots, e_{i}+(n-1) \pi_{k}, \ldots\right\}$

c) $S_{k}=\left\{q \pi_{k}+e_{i}: e_{i} \in C_{k}, q \in \mathbb{N} \cup\{0\}\right\}$, and is closed under multiplication.

Proof. Part a) simply states that, by the Partition Theorem, the equivalence relation of "congruence $\bmod \pi_{k}$ " partitions the initial set $S_{k}$ into equivalence classes which have the elements of $C_{k}$ as canonical representatives. Part b) follows from the definition of congruence mod $\pi_{k}$ in $S_{k}$. Finally, from a) and b), we see that $S_{k}$ can be obtained by adding successive multiples of $\pi_{k}$ to the elements of $C_{k}$. Furthermore, $\left(x, \pi_{k}\right)=1$ and $\left(y, \pi_{k}\right)=1$ iff $\left(x y, \pi_{k}\right)=1$ yields the closure of $S_{k}$ under multiplication.

Definition 2. If $x \in S_{k}$ and $t \in \mathbb{N}$, then the $t$-th multiple of $x$ in $S_{k}$ will be denoted by $\stackrel{(t)}{x}$. That is, $\stackrel{(t)}{x}=x s_{t}$, where $s_{t}$ is the $t$-th element of $S_{k}$. 
From now on we will refer to a multiple of an element $x$ in $S_{k}$, or to the $t$-th multiple of $x$ in $S_{k}$ by a simply saying a multiple of $x$ or the $t$-th multiple of $x$.

\section{MAIN RESULTS}

Proposition 3. Let $x \in S_{k}$. Any $m_{k}$ consecutive multiples of $x$ fall into different equivalent classes.

Proof. Let $x s_{i}, x s_{i+1}, \ldots, x s_{i+m_{k}-1}$ be a set of $m_{k}$ consecutive multiples of $x$. Proceeding by way of contradiction, suppose that $x s_{u}$, $x s_{v} \in\left[e_{t}\right]$ for some $e_{t} \in C_{k}$, and $u \neq v$, where $i \leq u, v \leq i+m_{k}-1$. Thus we have that $x s_{u}=q_{1} \pi_{k}+e_{t}$ and $x s_{v}=q_{2} \pi_{k}+e_{t}$, for some $q_{1}, q_{2} \in \mathbb{N} \cup\{0\}$. Without loss of generality, assume that $s_{u}>s_{v}$. Therefore,

$$
x\left(s_{u}-s_{v}\right)=\left(q_{1}-q_{2}\right) \pi_{k} .
$$

Since $x$ and $\pi_{k}$ have no common factors, this implies that $\pi_{k} \mid\left(s_{u}-s_{v}\right)$, which is a contradiction since by construction $\pi_{k}>s_{u}-s_{v}>0$.

Next we show that the multiples of any element $x \in S_{k}$ that appear within a given equivalence class can be found, like in the original Sieve, by counting every $x$ positions starting from the first multiple of $x$ in the class.

Theorem 4. The difference between the positions of two consecutive multiples of any element $x \in S_{k}$ within a given equivalence class is $x$.

Proof. If $x=1$, then we are done. So let $x \in S_{k}, x>1$, such that $\stackrel{(t)}{x} \in\left[e_{j}\right]$, for some $e_{j} \in C_{k}$ and $t \in \mathbb{N}$. We want to show that the next multiple of $x$ in $S_{k}$ that falls into the equivalence class $\left[e_{j}\right]$ is $\stackrel{(t)}{x}+x \pi_{k}$.

Let $\stackrel{(t)}{x}=q \pi_{k}+e_{j}$ for some $q \in \mathbf{N} \cup\{0\}$. Then,

$$
\stackrel{(t)}{x}+x \pi_{k}=\left(q \pi_{k}+e_{j}\right)+x \pi_{k}=(q+x) \pi_{k}+e_{j} \in\left[e_{j}\right] .
$$

Thus $\stackrel{(t)}{x}+x \pi_{k}$ is a multiple of $x$ in $\left[e_{j}\right]$. All that remains to be shown is that there does not exist a multiple of $x$, say $\stackrel{(m)}{x}$, such that $\stackrel{(m)}{x} \in\left[e_{j}\right]$ 
and $\stackrel{(t)}{x}<\stackrel{(m)}{x}<\stackrel{(t)}{x}+x \pi_{k}$. By way of contradiction, suppose that such an $\stackrel{(m)}{x}$ does exist in $\left[e_{j}\right]$. Thus, $\stackrel{(m)}{x}=u \pi_{k}+e_{j}$, for some $u \in \mathbb{N} \cup\{0\}$. Then from $\stackrel{(t)}{x}<\stackrel{(m)}{x}<x$

$$
q \pi_{k}+e_{j}<u \pi_{k}+e_{j}<(q+x) \pi_{k}+e_{j} \text {, and so } q<u<q+x \text {. }
$$

Now, $u=q+r$ for some $r \in \mathbf{N}$, where $1 \leq r<x$. Hence,

$$
\stackrel{(m)}{x}=(q+r) \pi_{k}+e_{j}=\left(q \pi_{k}+e_{j}\right)+r \pi_{k}=\stackrel{(t)}{x}+r \pi_{k} .
$$

Thus, $\stackrel{(m)}{x}-\stackrel{(t)}{x}=r \pi_{k}$ with $1 \leq r<x$. But $\left(x, \pi_{k}\right)=1$ and $x \mid(\stackrel{(m)}{x}-\stackrel{(t)}{x})$. This implies that $x \mid r$, which is a contradiction.

We have seen in theorem 4 that for any element $x \in S_{k}$, once we find its first multiple in an equivalence class, the remaining multiples are found by constant addition. By proposition 3, the first $m_{k}$ multiples of $x$ fall each into a different equivalence class. Hence, we have reduced to $m_{k}$ the number of products needed to determine all of the multiples of $x$. Our next result shows that the position within an equivalence class of any multiple of $x$ is totally dependent on the canonical representative of the equivalence class containing $x$. More precisely, once we have found the first multiple of $e_{j} \in C_{k}$ in some equivalence class [ $\left.e_{t}\right]$, then we can find all the multiples of $x \in\left[e_{j}\right]$ that fall in $\left[e_{t}\right]$ by adding some constant to the first multiple of $e_{j}$ in $\left[e_{t}\right]$.

Proposition 5. Let $x \in S_{k}$ be the $(q+1)$-th element in the equivalence class $\left[e_{i}\right]$, for some $e_{i} \in C_{k}$. If $\stackrel{(t)}{i}_{i}$ is the $v$-th element of some equivalence class $\left[e_{j}\right]$, then $\stackrel{(t)}{x}$ is the $\left(v+q s_{t}\right)$-th element of $\left[e_{j}\right]$, where $s_{t}$ is the $t$-th element of $S_{k}$.

Proof. Since $x$ is the $(q+1)$-th element in the equivalence class $\left[e_{i}\right]$, from Proposition $1 \mathrm{~b}$ ), we can write $x=q \pi_{k}+e_{i}$. Hence,

$$
\stackrel{(t)}{x}=\left(e_{i}+q \pi_{k}\right) s_{t}=e_{i} s_{t}+q s_{t} \pi_{k}=\stackrel{(t)}{e_{i}}+q s_{t} \pi_{k}=\left[(v-1) \pi_{k}+e_{j}\right]+q s_{t} \pi_{k} .
$$


But this says that $\stackrel{(t)}{x}=\left(v+q s_{t}-1\right) \pi_{k}+e_{j}$, and this is precisely what we want.

Corollary 6. If $x=(q+1) \pi_{k}+e_{i}$ and $e_{i} e_{t} \in\left[e_{j}\right]$ then $x e_{t}$, the first multiple of $x$ in $\left[e_{j}\right]$, is the $\left(q e_{t}\right)$-th element from $e_{i} e_{t}$ in $\left[e_{j}\right]$.

Proof. This is just a restatement of the former proposition in the particular case when $s_{t}=e_{t}$.

We have seen that the first $m_{k}$ multiples of the canonical representative of an equivalence class determines the positions of the first $m_{k}$ multiples of any element in the class. This suggests that, before initiating the sieving process on $S_{k}$, we should have available a table containing the products $e_{i} e_{j}$ for $i, j \in\left\{1,2, \ldots, m_{k}\right\}$.

Definition 7. We denote by $A$ the $m_{k} \times m_{k}$ array containing the products $e_{i} e_{j}$ for $1 \leq i, j \leq m_{k}$.

Notice that $A$ is symmetric, since both the $i$-th row and the $i$-th column of $A$ consist of the first $m_{k}$ multiples of $e_{i}$ and that the first row (column) consists of the elements of $C_{k}$. Hence, we only need to calculate the elements $a_{i j}$ for $2 \leq i \leq j \leq m_{k}$ of $A$. However, the number of calculations $m_{k}\left(m_{k}-1\right) / 2$ is still considerably large even for small values of $k$. Our next result shows that the number of calculations needed to obtain $A$ can be further reduced.

Theorem 8. Let $C_{k}=\left\{e_{1}, e_{2}, \ldots, e_{m_{k}}\right\}$ where $e_{i}<e_{j}$ for $i<j$. The following hold:

a) $\pi_{k}-e_{i} \in C_{k}$, for each $e_{i} \in C_{k}$,

b) $e_{i}+e_{j}=\pi_{k}$ iff $i+j=m_{k}+1$, and

c) $e_{j} e_{m_{k}+1-t}=\pi_{k} e_{j}-e_{j} e_{t}$ for $1 \leq j, t \leq m_{k}$.

Proof. a) We need to show that $\pi_{k}-e_{i}$ and $\pi_{k}$ are relatively prime. But if $p \mid\left(\pi_{k}-e_{i}\right)$ and $p \mid \pi_{k}$ for some prime $p$, then $p \mid\left[\pi_{k}-\left(\pi_{k}-e_{i}\right)\right]=e_{i}$, which is a contradiction to $e_{i} \in S_{k}$.

b) By hypothesis $e_{i}<e_{j}$ for $i<j$, thus it follows from a) that

$$
\pi_{k}-e_{m_{k}}<\pi_{k}-e_{m_{k}-1}<\ldots<\pi_{k}-e_{2}<\pi_{k}-e_{1},
$$


but this says that $e_{i}=\pi_{k}-e_{m_{k}-i}$ for $1 \leq i \leq m_{k}$, since $\pi_{k}-e_{i} \in C_{k}$.

c) Since $t+\left(m_{k}+1-t\right)=m_{k}+1$, it follows from b) that $e_{t}+$ $e_{m_{k}+1-t}=\pi_{k}$.

Corollary 9. $A$ is completely determined by the set

$$
T=\left\{e_{i} e_{j}: 2 \leq i \leq j \leq m_{k} / 2\right\}
$$

Proof. Consider the four $\left(\frac{m_{k}}{2} \times \frac{m_{k}}{2}\right)$ submatrices $B, C, D$ and $E$ of the matrix $A$ as depicted below. Clearly the first row of $A$ consists of the elements of $C_{k}$, since $e_{1}=1$.

Once the set $T$ is calculated, $B$ is completely known by symmetry. Using Theorem $8 \mathrm{c}$ ) the submatrix $C$ is immediately obtained from $B$. Moreover, the submatrix $D$ is then the reflection of $C$ upon the main diagonal of $A$. Finally, $E$ is obtained from $D$ by applying Theorem $8 \mathrm{c}$ ) again.

We remark that corollary 8 establishes that $A$ is completely determined by less than $\frac{1}{8}$ of its elements.

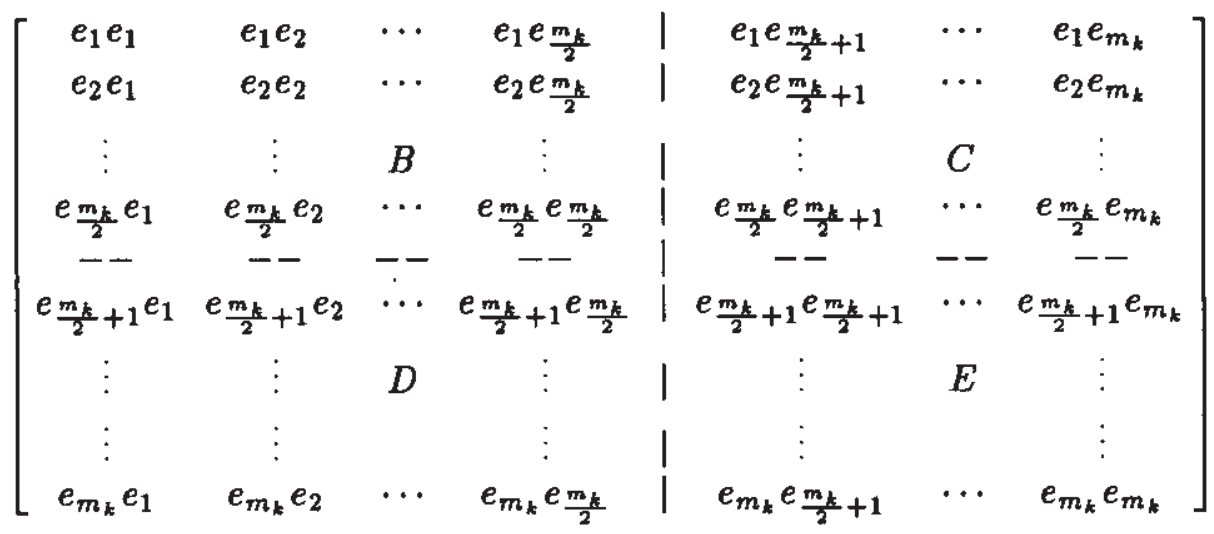

The following example illustrates our main results. 
Example 10. Let $k=3$. Then $\pi_{3}=2 \cdot 3 \cdot 5=30, C_{3}=$ $\{1,7,11,13,17,19,23,29\}$, and $m_{3}=\phi(30)=(2-1)(3-1)(5-1)=8$.

The set $T=\left\{7^{2}, 7 \cdot 11,7 \cdot 13,11^{2}, 11 \cdot 13,13^{2}\right\}$ contains the elements $b_{i j}$, where $1<i \leq j \leq 4$, of the submatrix $B$. The remaining elements of $B$ are found by symmetry. Theorem $8 \mathrm{c}$ ) yields $C$, since $c_{i j}=30 b_{i 1}-$ $b_{i(5-j)}, 1 \leq i, j \leq 4$.

$$
B=\left[\begin{array}{cccc}
1 & 7 & 11 & 13 \\
7 & 49 & 77 & 91 \\
11 & 77 & 121 & 143 \\
13 & 91 & 143 & 169
\end{array}\right], C=\left[\begin{array}{cccc}
17 & 19 & 23 & 29 \\
119 & 133 & 161 & 203 \\
187 & 209 & 253 & 319 \\
221 & 247 & 299 & 377
\end{array}\right]
$$

By symmetry on $A$ we obtain $D$, i.e., $d_{i j}=c_{j i}, 1 \leq i, j \leq 4$. Finally, $E$ is obtained from $D$ by applying Theorem $8 \mathrm{c}$ ) again.

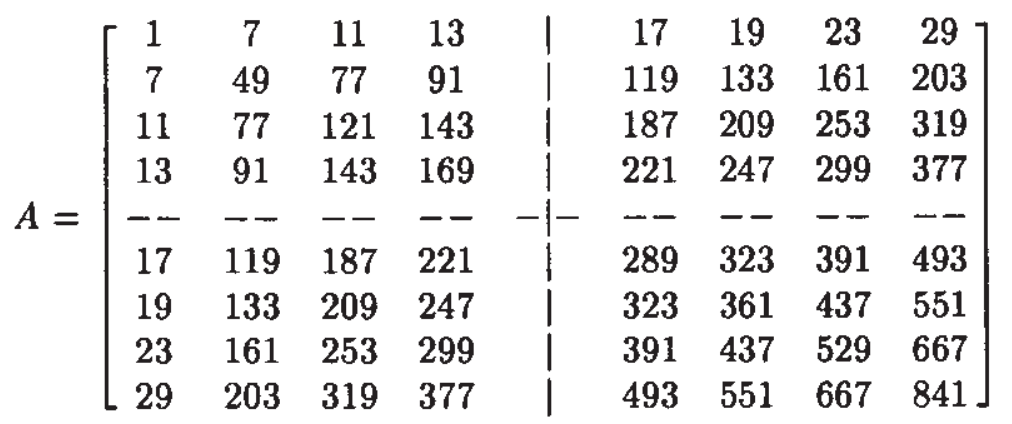

Using the characterization of Proposition $1 \mathrm{c}$ ) we obtain $S_{3}$ by adding multiples of 30 to $C_{3}$. Thus, as depicted in Table 1, we may express $S_{3}$ as an infinite array where each column is an equivalence class whose canonical representative is the corresponding element of $C_{3}$ in the first row.

The second row of $A$ contains the first eight multiples of 7 in $S_{3}$, each in a different column, which we have parenthesized in the table. To delete the remaining multiples of 7 in an equivalence class, say those in [13], we locate the first multiple of 7 in [13] which is 133 . Then we cancel every 7 -th element after 133 in that column. The first eight multiples of 
a prime number in the $i$-th row of table 1 and in the equivalence class [7], can be easily obtained by adding $30(i-1) e_{1 j}$ to $a_{2 j}, 1 \leq j \leq 8$. Thus, the first eight multiples of 67 are $60+7,420+49, \ldots, 1740+203$. We have also underlined the multiples of 11 in the table.

We remark that after $A$ is obtained, the sieving of $S_{k}$ becomes an additive process. Moreover, the deletion of the multiples of any prime in different columns can be done independently. Because of these two reasons, this is a fast algorithm particularly suited for parallel implementation.

$\begin{array}{cccccccc}{[1]} & {[(7)]} & {[\underline{11}]} & {[13]} & {[17]} & {[19]} & {[23]} & {[29]} \\ 31 & 37 & 41 & 43 & 47 & (49) & 53 & 59 \\ 61 & 67 & 71 & 73 & (\underline{77}) & 79 & 83 & 89 \\ (91) & 97 & 101 & 103 & 107 & 109 & 113 & (119) \\ \underline{121} & 127 & 131 & (133) & 137 & 139 & \underline{143} & 149 \\ 151 & 157 & (161) & 163 & 167 & 169 & 173 & 179 \\ 181 & \underline{187} & 191 & 193 & 197 & 199 & (203) & \underline{209} \\ 211 & (217) & 221 & 223 & 227 & 229 & 233 & 239 \\ 241 & 247 & 251 & \underline{253} & 257 & (259) & 263 & 269 \\ 271 & 277 & 281 & 283 & (287) & 289 & 293 & 299 \\ (301) & 307 & 311 & 313 & 317 & \underline{319} & 323 & (329) \\ 331 & 337 & \underline{341} & (469) & 347 & 349 & 353 & 359 \\ 361 & 367 & (371) & 373 & 377 & 379 & 383 & 389 \\ 391 & 397 & 401 & 403 & 407 & 409 & (413) & 419 \\ 421 & (427) & 431 & 433 & 437 & 439 & 443 & 449 \\ \underline{451} & 457 & 461 & 463 & 467 & (469) & \underline{473} & 479 \\ . & . & . & . & . & . & . & . \\ . & . & . & . & . & . & . & . \\ . & . & . & . & . & . & . & .\end{array}$

Table 1

\section{References}

[1] Bengelloun, S.A., An incremental primal sieve, Acta Informatica 23, (1986), 119-125. 
[2] Mairson, H.G., Some new upper bounds on the generation of prime numbers, Commun. ACM 20, 9 (Sept. 1977), 664-669.

[3] Knuth, D., The Art of Computer Prognamming, Vol. 2, 2nd ed., p. 394, Addison Wesley. Publishing Company, Reading, Massachusetts, 1981.

[4] Pritchard, P., A sublinear additive sieve for finding prime numbers, Commun. ACM 24, 1 (Jan. 1981), 18-23.

[5] Quesada, A.R., Third Extension of Erathostenes' Sieve. Commun. $A C M$ 35, 3 (Mar. 1992), pp. 11-13.

[6] Quesada, A.R., On the $k$-th extension of the sieve of Eratosthenes. Int. J. of Math. and Math. Sci., Vol. 18, No. 3, pp. 539-544, 1995.

[7] Xuedong Luo, A practical sieve algorithm for finding prime numbers, Commun. ACM 32, 3 (Mar. 1989), 344-346. 